\title{
INTERFERENCIA DE Cenchrus echinatus Y Rottboellia exaltata EN EL CRECIMIENTO DEL FRIJOL CAUPÍ
}

\author{
Sonicley da Silva Maia ${ }^{1}$, Paulo R. Ribeiro-Rocha ${ }^{1}$, Thaís Santiago-Castro ${ }^{1}$, Iasmin K. A. Costa \\ da Silva ${ }^{1}$, Glauber Ferreira-Barreto ${ }^{1}$, Leandro Torres de Souza ${ }^{1}$, \\ Hipólito Murga-Orrillo² y Carlos Abanto-Rodríguez ${ }^{2}$
}

\begin{abstract}
RESUMEN
El frijol caupí es un cultivo importante para la seguridad alimentaria de la población mundial; sin embargo, su producción es afectada constantemente por la interferencia de las malezas. El objetivo de esta investigación fue determinar el efecto de la interferencia de las malezas en diferentes densidades de competencia sobre las características de crecimiento del cultivo. El estudio fue conducido mediante diseño de bloques al azar en arreglo factorial 2x4 para análisis del cultivo y 2x3 para las malezas. Fueron estudiadas dos especies de maleza (Cenchrus echinatus y Rottboellia exaltata) y cuatro intensidades de competencia (cero, dos, cuatro y seis individuos por maceta), con cuatro repeticiones. Las evaluaciones fueron realizadas en la etapa de floración del cultivo y las variables medidas fueron área foliar, masa seca de las hojas, del tallo, de la raíz (MSR), de la parte aérea (MSPA), masa seca total y la relación MSR/MSPA. Además, fueron cuantificadas en las malezas la masa seca de la parte aérea (MSPAM) y de la raíz (MSRM). El aumento de la densidad de competencia de las malezas resultó en disminución de los valores de todas las variables analizadas en el cultivo. En las malezas, $C$. echinatus mostró mayor MSPAM que R. exaltata, aunque no hubo diferencias en MSRM. Ambas malezas afectan negativamente el crecimiento de las plantas de frijol, aunque con $C$. echinatus el efecto es aún mayor. El efecto de interferencia es mayor a medida que aumenta la densidad de las malezas.

Palabras clave adicionales: Competencia biológica, población de plantas, Vigna unguiculata
\end{abstract}

\begin{abstract}
Interference of Cenchrus echinatus and Rottboellia exaltata in growth of cowpea crop

Cowpea is an important crop for the food security of the world population. However, their production is constantly affected by weed interferences. The objective of this research was to determine the effect of two different weeds at different densities of competition on the growth characteristics of the crop. The study was conducted using a randomized block design in a factorial arrangement of the treatments (2x4 for analysis in cowpea, and 2x3 for weeds). The weed species (Cenchrus echinatus and Rottboellia exaltata) and four competition densities (zero, two, four, and six individuals per pot), with four repetitions, were studied at the crop flowering stage. We measured leaf area, leaf and stem dry mass, root dry mass (RDM), dry mass of the aerial part (DMAP), total dry mass, and the ratio (RDM/DMAP); additionally, the dry mass of the aerial part and root (DMAW and DMRW) of the weeds was measured. The increase in the density of the weeds resulted in a decrease in the values of all the variables of the crop. In the case of weeds, $C$. echinatus showed higher DMAW than $R$. exaltata although there were no differences in DMRW. Both weeds negatively affect the growth of bean plants, though with C. echinatus the effect is even greater. The interference effect is higher as the density of the weeds increases.
\end{abstract}

Additional keywords: Biological competition, plant population, Vigna unguiculata, weeds

\section{INTRODUCCIÓN}

El frijol caupí (Vigna unguiculata (L.) Walp.) conocido popularmente como frijol de cuerda, frijol fradiño o frijol de rama, es cultivado en las regiones norte y noreste de Brasil principalmente por sus características nutritivas, por presentar alto contenido de proteína y en menor cantidad lípidos, polisacáridos, calcio, hierro, potasio, fósforo y aminoácidos esenciales (Silva et al., 2016).

Recibido: Abril 25, 2020

Aceptado: Noviembre 26, 2020

${ }^{1}$ Universidade Federal de Roraima UFRR, Centro de Ciências Agrárias, Boa Vista, Roraima, Brasil. e-mail: sony_maia@hotmail.com (autor de correspondencia); paulo.rocha@ufrr.br; thaiscastro.agr@gmail.com; iasmin.kele5@gmail.com; glauberfbarreto@gmail.com; leandro.torres@ufrr.br

${ }^{2}$ Universidad Nacional Autónoma de Chota UNACH, Chota, Cajamarca, Perú. e-mail: leohmurga@gmail.com; cabanto@unach.edu.pe 
Sin embargo, este cultivo presenta baja productividad cuando se compara con el potencial de la especie, la cual puede producir hasta 6.000 $\mathrm{kg} \cdot \mathrm{ha}^{-1}$ (Alves et al., 2009). En Brasil, la productividad media en la época de mayores cosechas del 2018-2019 alcanzó $491 \mathrm{~kg} \cdot \mathrm{ha}^{-1}$; la región norte obtuvo un rendimiento promedio de $994 \mathrm{~kg} \cdot \mathrm{ha}^{-1} \mathrm{y}$ los estados que se destacaron fueron Tocantins, Pará, Roraima y Acre (CONAB, 2019).

Este bajo rendimiento es resultado, entre otras posibles causas, del uso incipiente de tecnología en la labranza, de la baja calidad de las semillas, problemas fitosanitarios y competencia con las malezas. Esta competencia se convierte en un problema para cultivos de ciclo corto, pues, interfieren directamente en el desarrollo vegetativo y productivo al competir por agua, luz y nutrientes (Lamego et al., 2011; Pessôa et al., 2015). Al mismo tiempo, las malezas pueden liberar sustancias alelopáticas o ser hospederas de plagas, enfermedades, además de obstaculizar la cosecha (Pitelli, 1985).

Las pérdidas en la producción de frijol caupí causadas por interferencia de las malezas son reflejadas principalmente en la disminución del número de vainas, masa de los granos y productividad (Oliveira et al., 2010). De esta manera, Freitas et al. (2009) constataron que las malezas cuando no son controladas en las labores culturales pueden reducir el rendimiento en hasta $90 \%$ de granos del frijol.

Algunas especies de malezas presentan mayor competitividad con los cultivos debido a su eficiencia en la absorción y utilización de nutrientes (Di Tomaso, 1995). En ese sentido, Cury et al. (2011) señalan que las especies Cenchrus echinatus y Bidens pilosa presentaron elevada competencia, siendo capaces de provocar reducciones significativas en la acumulación de masa seca del frijol común (Phaseolus vulgaris L.).

Por su parte, en condiciones de sabana, Albuquerque et al. (2013) observaron en el cultivo de maíz, que los mayores índices de valor de importancia (IVI) encontrados en el área fueron para las especies C. echinatus, Sida rhombifolia, Digitaria sanguinalis y Cyperus rotundus. Recientemente, Castro et al. (2019) determinaron que las malezas Rottboellia exaltata, Digitaria horizontalis y C. echinatus causaron daños en la productividad del cultivo de frijol debido a su alto poder competitivo, a causa del rápido desarrollo y sincronización con la fase reproductiva del frijol.

Para las condiciones de sabana de Roraima, en el extremo norte del Brasil, existe poca información del efecto de malezas sobre el cultivo de frijol caupí, a la vez que el paquete tecnológico del cultivo en este estado continúa en fase de creación y validación. Por lo tanto, el objetivo en este trabajo fue determinar el efecto de la interferencia de malezas con cuatro densidades de competencia sobre las características de crecimiento del frijol.

\section{MATERIALES Y MÉTODOS}

El experimento fue conducido en ambiente protegido (casa de cultivo), con estructura metálica tipo tijera, sobre la cual fueron fijadas dos capas de policarbonato transparente, con paredes laterales constituidas del mismo material transparente de $1 \mathrm{~cm}$ de espesor. La temperatura y la humedad relativa del aire fueron controladas usando un sistema de ventilación forzada $y$ presentaron valores medios durante el experimento de $30 \pm 2{ }^{\circ} \mathrm{C}$ y $70 \%$, respectivamente. Esta estructura se encuentra dentro del área experimental del Centro de Ciencias Agrarias de la Universidad Federal de Roraima - CCA/UFRR, en el municipio de Boa Vista, Roraima, Brasil, localizado en las coordenadas $2^{\circ} 52^{\prime} \mathrm{N}, 60^{\circ} 42^{\prime} \mathrm{W}$ y 90 m de altitud.

El suelo utilizado fue clasificado como Geric Xanthic Ferralsol, el cual fue recolectado en la misma área experimental a una profundidad de 0$10 \mathrm{~cm}$, tamizado en malla de $5 \mathrm{~mm}$; sus características químicas se presentan en el Cuadro 1. Para la adecuación del suelo en cuanto a la fertilización fue aplicada cal dolomítica para elevar la saturación de base al 60 \% y el suelo fue entonces incubado por 30 días. Luego de ese período fue aplicado por $\mathrm{m}^{3}$ de suelo $0,2 \mathrm{~kg}$ de $\mathrm{N}$, $1,8 \mathrm{~kg}$ de $\mathrm{P}_{2} \mathrm{O}_{5}, \mathrm{y} 0,203 \mathrm{~kg}$ de $\mathrm{K}_{2} \mathrm{O}$, teniendo como fuentes urea, superfosfato simple y cloruro de potasio, respectivamente; posteriormente se adicionaron micronutrientes (fertilizante FTE BR12) a razón de $50 \mathrm{~g} \cdot \mathrm{m}^{3}$ de suelo, siguiendo la recomendación de Novais (2002).

El diseño experimental fue de bloques completamente al azar, con arreglo factorial $2 \times 4$, conformado por dos especies de malezas y cuatro densidades de competencia sobre frijol (cero, dos, cuatro y seis individuos por unidad experimental), 
con cuatro repeticiones. Para el análisis de las variables referentes a las malezas, el factorial fue $2 \mathrm{x} 3$, con dos especies de malezas $\mathrm{y}$ tres densidades. Las unidades experimentales fueron constituidas de macetas cónicas de polietileno con $24 \mathrm{~cm}$ de diámetro superior y capacidad de $8 \mathrm{~L}$.

Cuadro 1. Características químicas del suelo utilizado área experimental del Centro de Ciencias Agrarias UFRR, en Boa Vista, Roraima

\begin{tabular}{cccccccccc}
\hline $\mathrm{pH}$ & $\begin{array}{c}\mathrm{MO} \\
\mathrm{g} \cdot \mathrm{dm}^{-3}\end{array}$ & $\begin{array}{c}\mathrm{P} \\
\mathrm{mg} \cdot \mathrm{dm}^{-3}\end{array}$ & $\begin{array}{c}\mathrm{K} \\
-----\mathrm{Ca}^{2+}\end{array}$ & $\begin{array}{c}\mathrm{Mg}^{2+} \\
\mathrm{Al}^{3+}\end{array}$ & $\mathrm{H}+\mathrm{Al}$ & $\mathrm{CIC}$ & $\mathrm{SB}$ \\
\hline 5,4 & 5,00 & 3,00 & 0,08 & 0,90 & 0,60 & 0,10 & 2,20 & 3,78 & 41,80 \\
\hline
\end{tabular}

pH en agua (1:2:5); MO: materia orgánica; $\mathrm{Ca}^{2+}, \mathrm{Mg}^{2+}$ y $\mathrm{Al}^{3+}$ : extractor $\mathrm{KCl} 1 \mathrm{~mol} \mathrm{~L}^{-1} ; \mathrm{K}^{+}$y P: extractor Mehlich-1; $\mathrm{H}+\mathrm{Al}$ : extractor acetato de calcio; CIC: capacidad de intercambio catiónico; SB: saturación por bases

En lo relativo al material vegetal, fueron seleccionadas plántulas de las malezas $C$. echinatus, de hábito rastrero, y $R$. exaltata, de crecimiento erecto; ambas pertenecen a la familia Poaceae, presentan crecimiento rápido y son comúnmente encontradas compitiendo con diversos cultivos. Las semillas de frijol utilizadas fueron del cultivar BRS Guariba, que presenta hábito de crecimiento indeterminado, porte semierecto, con floración a los 41 días y ciclo de 65-70 días después de la siembra (EMBRAPA, 2009).

En el centro de cada maceta fueron sembradas dos semillas de frijol, a profundidad media de $2 \mathrm{~cm}$. A loe 5 días después de la emergencia fue realizado el entresaque, dejando una plántula por maceta. Por otra parte, las malezas previamente identificadas y recolectadas de campos de cultivo, se trasplantaron a las macetas en el área periférica de las plántulas de frijol, conformando las cuatro densidades de competencia de las malezas. Para la sincronización de las malezas con las plántulas de frijol el trasplante fue realizado cuando las especies presentaron dos hojas verdaderas.

Los tratamientos fueron mantenidos bajo riego por microaspersión, dejando el suelo con humedad próxima de la capacidad de campo. A los 16 días después de la siembra se realizó una fertilización complementaria utilizando urea como fuente de $\mathrm{N}$, en dosis equivalente a $20 \mathrm{~kg} \cdot \mathrm{ha}^{-1}$.

Las evaluaciones fueron efectuadas cuando las plantas de frijol estaban en plena floración, lo que ocurrió a los 37 días de interacción de las malezas y el cultivo. Para esto se colectaron todas las plantas de las macetas; en las plantas de frijol se separaron el tallo, las hojas y las raíces; en hojas separadas se determinó el área foliar (AF) del cultivo utilizando un equipo Li-Cor 3100. En las malezas se separó la raíz y la parte aérea.

Seguidamente, los tallos, hojas y raíz del frijol, así como la parte aérea y las raíces de las malezas fueron acondicionadas en bolsas de papel y llevadas a estufa de circulación forzada, a temperatura de $70{ }^{\circ} \mathrm{C}$ hasta alcanzar peso constante. Después del secado fue determinada la masa seca de las hojas (MSH), del tallo (MST) y de la raíz (MSR). A partir de estas variables fueron calculadas la masa seca total del frijol $($ MSTo $=$ MSH+MST+MSR $)$ y la relación de la masa seca de raíz y masa seca de la parte aérea del frijol (MSR/MSPA). Para las malezas fueron determinadas la masa seca de la parte aérea (MSPAM) y la masa seca de raíz (MSRM). Para todas estas variables fue utilizada una balanza con apreciación de $0,01 \mathrm{~g}$.

Los resultados de las variables agronómicas del cultivo fueron analizados mediante pruebas de regresión para los niveles de densidades de las malezas, escogiendo los modelos de mejor ajuste en función de sus coeficientes de determinación $\left(\mathrm{R}^{2}\right)$. Los pesos de la materia seca aérea y radical de las malezas fueron sometidos a análisis de variancia y prueba de Tukey. Para todos los análisis se utilizó el software Sisvar (Ferreira, 2011).

\section{RESULTADOS Y DISCUSIÓN}

El análisis de varianza (Cuadro 2) muestra que para las variables relacionadas al frijol hubo efecto significativo en la interacción entre maleza y densidad de competencia para la variable AF y efecto simple en densidad de competencia para las variables MSH, MST, MSR, MSTo y MSR/MSPA.

Para las variables relacionadas a las malezas, no hubo efecto significativo de la interacción en 
las variables analizadas. No obstante, hubo efecto aislado de los factores evaluados para MSPAM y efecto simple del factor densidad para la MSRM (Cuadro 2).

El AF de las plantas de frijol presentó reducción con el aumento de las densidades de la especie C. echinatus, a mayor densidad de la especie se observó una reducción de 39 \% cuando se comparó con el frijol del tratamiento control (Figura 1). Por su parte, la mayor reducción alcanzada con $R$. exaltata fue de solo $18 \%$.

Estos resultados deben estar relacionados con los hábitos de crecimiento de las malezas, pues $C$. echinatus tiene hábito rastrero, con capacidad de multiplicarse mediante estolones, proporcionando rápidamente mayor competencia, y consecuentemente habría disminuido el espacio, nutrientes y humedad disponible para el cultivo. Por otro lado, $R$. exaltata posee crecimiento erecto, con multiplicación predominantemente por semilla lo que proporciona un desarrollo menos rápido comparado con C. echinatus.

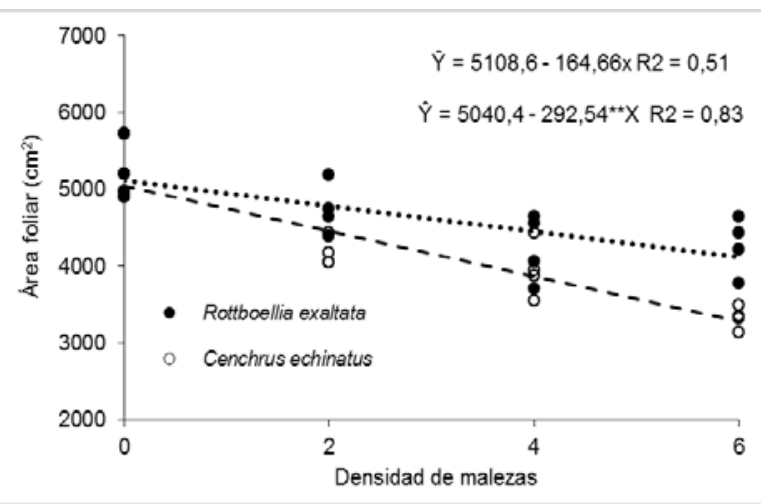

Figura 1. Área foliar (AF) del frijol en función de cuatro densidades de dos malezas, después de 37 días de competencia

Cuadro 2. Resumen de los análisis de varianza de las variables agronómicas del cultivo y de las masas secas de la parte aérea y raíz de las malezas en función de sus diferentes densidades después de 37 días de competencia

\begin{tabular}{|c|c|c|c|c|c|c|c|}
\hline \multirow{3}{*}{ Fuente de Variación } & \multirow{3}{*}{ GL } & \multicolumn{6}{|c|}{ Cuadrados Medios } \\
\hline & & $\mathrm{AF}\left(\mathrm{m}^{2}\right)$ & MSH (g) & MST (g) & MSR (g) & MSTo (g) & MSR/MSPA $\left(g \cdot g^{-1}\right)$ \\
\hline & & \multicolumn{6}{|c|}{ Frijol } \\
\hline Maleza (M) & 1 & $3238722,47^{* *}$ & $4,62 \mathrm{~ns}$ & $3,11 \mathrm{~ns}$ & $0,09 \mathrm{~ns}$ & $17,72 \mathrm{~ns}$ & $0,000000 \mathrm{~ns}$ \\
\hline Densidad (D) & 3 & $2827937,50^{* *}$ & $69,02^{* *}$ & $27,71^{* *}$ & $10,32^{* *}$ & $272,48^{* *}$ & $0,004054^{* *}$ \\
\hline$M \times D$ & 3 & $833345,96^{*}$ & $15,85 \mathrm{~ns}$ & $2,79 \mathrm{~ns}$ & $0,15 \mathrm{~ns}$ & $21,20 \mathrm{~ns}$ & $0,000858 \mathrm{~ns}$ \\
\hline Bloque & 3 & 627200,03 ns & $9,05 \mathrm{~ns}$ & 2,97 ns & $0,28 \mathrm{~ns}$ & $23,29 \mathrm{~ns}$ & $0,000621 \mathrm{~ns}$ \\
\hline Error & 21 & 239088,44 & 9,37 & 4,08 & 0,35 & 25,98 & 0,000357 \\
\hline \multirow[t]{3}{*}{$\mathrm{CV} \%$} & & 11,23 & 24,06 & 14,97 & 24,06 & 17,79 & 20,35 \\
\hline & & \multicolumn{6}{|c|}{ Malezas } \\
\hline & & \multicolumn{3}{|c|}{ MSPAM (g) } & \multicolumn{3}{|c|}{ MSRM (g) } \\
\hline Maleza (M) & 1 & \multicolumn{3}{|c|}{$80,520067^{*}$} & \multicolumn{3}{|c|}{$0,142604 \mathrm{~ns}$} \\
\hline Densidad (D) & 2 & \multicolumn{3}{|c|}{$218,243338^{* *}$} & \multicolumn{3}{|c|}{$18,776704^{* *}$} \\
\hline$M \times D$ & 2 & \multicolumn{3}{|c|}{$14,876654 \mathrm{~ns}$} & \multicolumn{3}{|c|}{$0,030929 \mathrm{~ns}$} \\
\hline Bloque & 3 & \multicolumn{3}{|c|}{$6,840139 \mathrm{~ns}$} & \multicolumn{3}{|c|}{ 0,340193 ns } \\
\hline Error & 15 & \multicolumn{2}{|c|}{11,924172} & & \multicolumn{3}{|c|}{0,442030} \\
\hline $\mathrm{CV} \%$ & 35 & \multicolumn{2}{|c|}{26,31} & & \multicolumn{3}{|c|}{18,25} \\
\hline
\end{tabular}

AF: área foliar, MSH: masa seca de las hojas, MST: masa seca del tallo, MSR: masa seca de la raíz, MSTo: masa seca total, MSR/MSPA: relación de las masas secas de raíz y parte aérea del frijol; MSPAM: masa seca de la parte aérea de las malezas, MSRM: masa seca de la raíz de las malezas. GL: grados de libertad; CV: coeficiente de variación; ns, * y **: no significativo, significativo al $5 \%$ y significativo a $1 \%$, respectivamente, mediante la prueba de $\mathrm{F}$

La disminución del AF, como consecuencia de la mayor competencia de malezas, puede afectar negativamente los procesos fotosintéticos de las plantas, lo que ocasiona pérdidas significativas en la productividad de frijol (Oliveira et al., 2015). Estos efectos también fueron encontrados por Forte et al. (2017) cuando evaluaron la influencia de diferentes densidades de B. pilosa y Euphorbia heterophylla sobre dos cultivares de soja y observaron que los aumentos de las densidades de estas especies tuvieron incidencia inversamente proporcional al desarrollo foliar del cultivo.

Similarmente a lo ocurrido con el AF, a medida que aumentó la densidad de las malezas 
hubo disminución en los valores de masa seca de las hojas del frijol, en comparación con el tratamiento control (Figura 2).

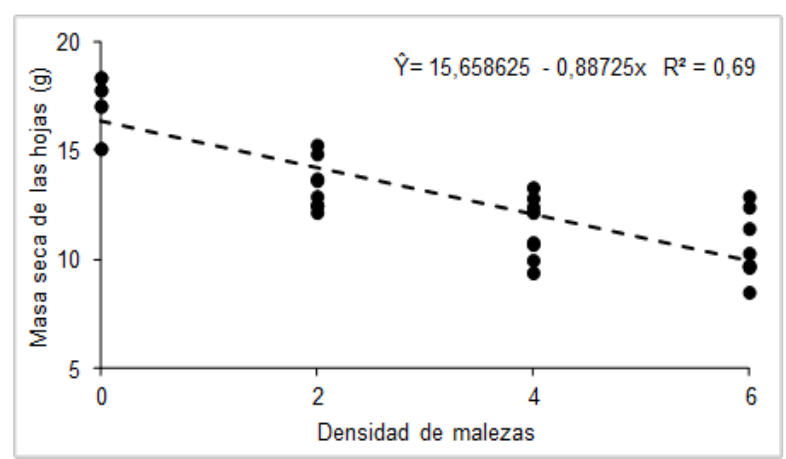

Figura 2. Masa seca de las hojas (MSH, g) del frijol en función de cuatro densidades de dos malezas, después de 37 días de competencia

Resultados similares fueron encontrados por Manabe et al. (2014) cuando evaluaron la influencia de B. pilosa, y por Lage et al. (2017) en un ensayo de competencia de Brachiaria plantaginea y B. pilosa, ambos sobre el desarrollo foliar de frijol común, y determinaron que, por el aumento de las densidades de competencia, hubo reducción en los valores de MSH.

En nuestro estudio el aumento de las densidades de las dos especies de maleza también disminuyó el desarrollo del tallo de las plantas de frijol y la mayor densidad promovió la reducción de alrededor del $27 \%$ de la MST del frijol, en comparación el tratamiento control (Figura 3).

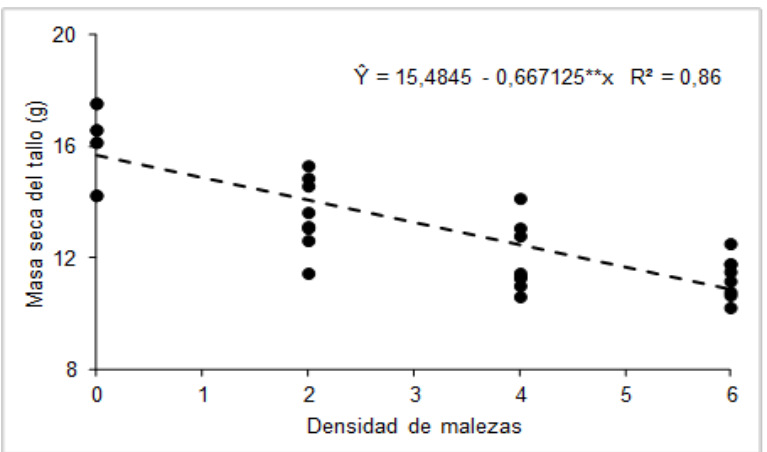

Figura 3. Masa seca del tallo (MST, g) del frijol en función de cuatro densidades de dos malezas, después de 37 días de competencia

La reducción de MST también ha sido observada por Manabe et al. (2015) después que evaluaron tres especies de malezas sobre el desarrollo del cultivo del frijol, determinando que $B$. pilosa fue la especie que más influyó en la reducción del peso seco del tallo del cultivo.

Para la MSR de las plantas de frijol se observa que después de 37 días de convivencia, en la densidad de seis malezas por maceta, hubo reducción de $65 \%$, comparando con el tratamiento control (Figura 4).

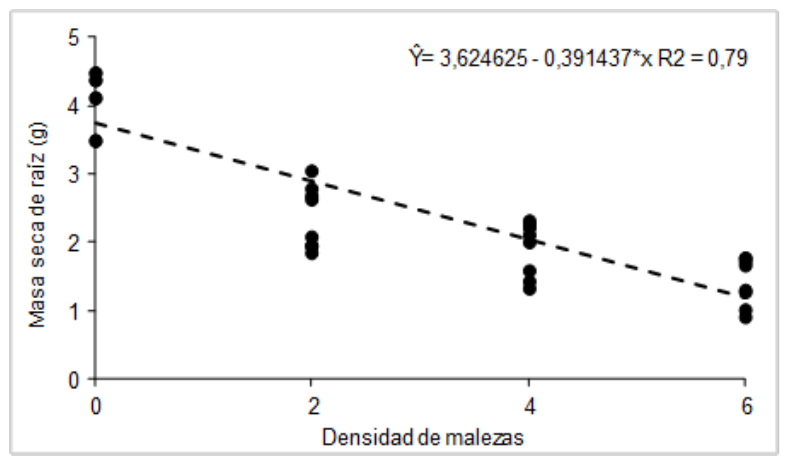

Figura 4. Masa seca de raíz (MSR, g) del frijol en función de cuatro densidades de dos malezas, después de 37 días de competencia

En la reacción a sobrevivir a la competencia, es posible suponer que el potencial agresivo de una maleza a nivel radicular hace que el cultivo principal induzca a la mayor asignación de masa seca a los órganos superiores (Cury et al. 2013; Manabe et al. 2015), entendiéndose que el sistema radical es el principal órgano afectado por la competencia. La reducción del sistema radicular puede implicar menor tolerancia al déficit hídrico y menor capacidad de absorción de nutrientes debido a la menor exploración del suelo por las raíces.

La MSTo del frijol se redujo con el aumento de las densidades de las malezas, con reducción media de $36 \%$ con la densidad de seis plantas por maceta, cuando fue comparado con el control (Figura 5).

El comportamiento de reducción de MSTo del frijol está relacionado con la disminución de los componentes que la conforman, es decir MSH, MST y MSR, los mismos que también se redujeron con el aumento de las densidades. Esto demuestra que cada especie de maleza tiene su potencial de competencia.

Lo anterior se corrobora con los resultados de Cury et al. (2011), quienes evaluaron la competencia de seis malezas diferentes en tres 
genotipos de frijol común y encontraron reducciones variadas de MSTo de estos cultivares por efecto de la competencia.

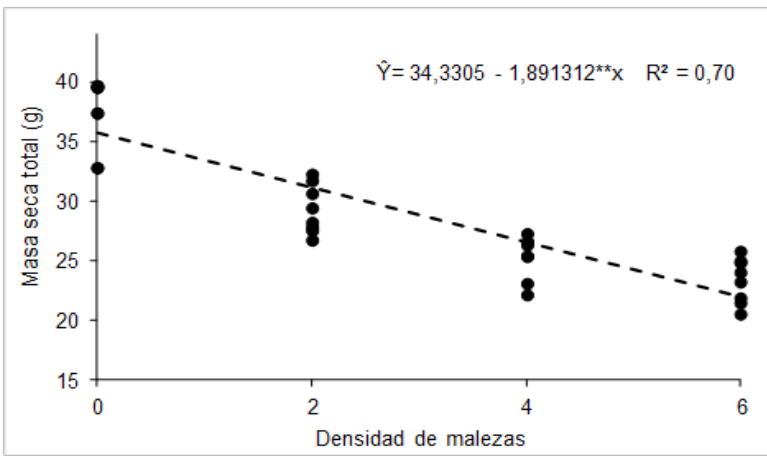

Figura 5. Masa seca total (MSTo, g) del frijol en función de cuatro densidades de dos malezas, después de 37 días de competencia

Al evaluar la relación de la MSR/MSPA de las plantas de frijol se encontró que hubo una reducción lineal con el aumento en la densidad, la cual interfirió en aproximadamente el $46 \%$ de esta variable cuando las plantas del frijol convivieron los 37 días de competencia con la densidad de seis plantas por maceta (Figura 6).

La reducción lineal de la relación MSR/MSPA de las plantas de frijol, cuando la planta competidora fue $R$. exaltata, está relacionado con la reducción de la MSR del cultivo, debido al aumento de la densidad de esa maleza, como se observa en la Figura 4.

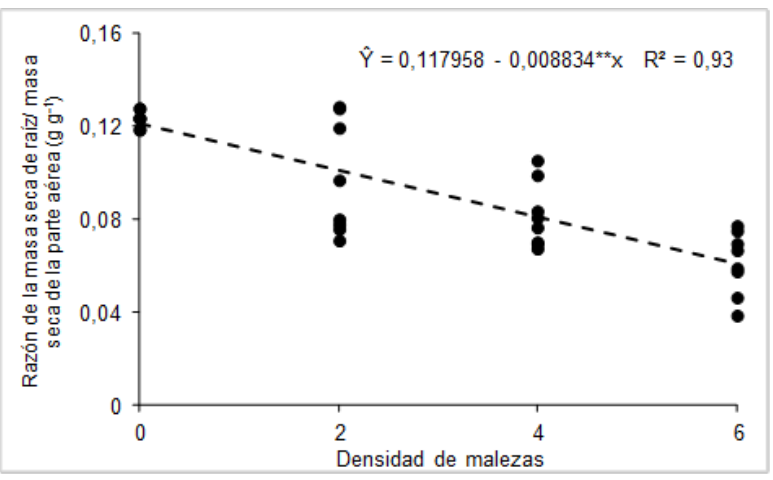

Figura 6. Relación de la masa seca de raíz/ masa seca de la parte aérea (MSR/MSPA) del frijol en función de cuatro densidades de malezas, después de 37 días de competencia

Los resultados de esa variable son semejantes a los obtenidos por Pessôa et al. (2015), que evaluaron diferentes poblaciones de C. rotundus y encontraron que la presencia de dos o más tubérculos de esta maleza ocasionó efecto negativo en la relación MSR/MSPA del frijol.

En relación con las variables de las malezas, se observa en el Cuadro 3 que $C$. echinatus difiere estadísticamente de $R$. exaltata, y presentó mayor valor medio de MSPAM. Sin embargo, para la MSRM no hubo diferencia significativa entre ambas especies de malezas.

Se destaca que $C$. echinatus presentó mayor media de la MSPAM, a pesar de no presentar diferencia significativa individualmente en las densidades estudiadas; sin embargo, fue esa maleza la que más influenció negativamente en las características de crecimiento del cultivo de frijol. De acuerdo a Parreira et al. (2011) el frijol tiene la capacidad de convivir con C. echinatus sin afectar significativamente su desarrollo, por un período de hasta 26 días después de la emergencia, correspondiendo este lapso a los estadios fenológicos que anteceden a la floración.

Para $R$. exaltata, se observa en el Cuadro 3 que esta maleza vio afectado su crecimiento en la competencia con el frijol, debido a que presenta desarrollo inicial lento, alcanzando la media de 11,29 g de MSPAM, indicando que no fue la especie que más perjudicó el crecimiento del frijol hasta la fase de pleno florecimiento.

La acumulación de masa seca, tanto de la parte aérea como de la raíz de las malezas, varió en las diferentes densidades. Se observa que, para ambas variables, la densidad de seis plantas por maceta fue la que obtuvo los mayores valores, seguido por las densidades de cuatro y dos plantas por maceta (Cuadro 3), indicando así que el ambiente donde ocurrió la competencia, hasta el período evaluado, proporcionó agua, luz y nutrientes suficientes para las especies dañinas, aún con el aumento de las densidades, aumentaron los valores medios de la MSPAM y MSRM.

Resultados similares fueron obtenidos por Terceiro et al. (2016) cuando evaluaron diferentes poblaciones de $C$. rotundus en el cultivo del frijol y determinaron que el mayor valor de MSPAM ocurrió en el tratamiento con mayor densidad (3 tubérculos por maceta), evidenciando que el aumento de las densidades de las malezas puede poseer una notable capacidad de producción de fitomasa. 
Cuadro 3. Masa seca de la parte aérea (MSPAM) y de la raíz de malezas (MSRM) por maceta, en las diferentes densidades, después de 37 días de competencia con el frijol

\begin{tabular}{|c|c|c|c|c|c|c|}
\hline \multirow{2}{*}{ Densidad } & \multicolumn{2}{|c|}{ MSPAM (g) } & \multirow{2}{*}{ Media } & \multicolumn{2}{|c|}{ MSRM (g) } & \multirow{2}{*}{ Media } \\
\hline & C. echinatus & R. exaltata & & C. echinatus & R. exaltata & \\
\hline 2 & 7,60 & 7,05 & $7,32 \mathrm{~b}$ & 2,13 & 2,27 & $2,20 \mathrm{c}$ \\
\hline 4 & 17,01 & 12,18 & $14,60 \mathrm{a}$ & 3,45 & 3,49 & $3,47 \mathrm{~b}$ \\
\hline 6 & 20,26 & 14,64 & $17,45 \mathrm{a}$ & 5,11 & 5,39 & $5,25 \mathrm{a}$ \\
\hline Media & $14,96 \mathrm{~A}$ & $11,29 \mathrm{~B}$ & & $3,56 \mathrm{~A}$ & $3,72 \mathrm{~A}$ & \\
\hline CV (\%) & \multicolumn{2}{|c|}{18,93} & \multicolumn{4}{|c|}{20,52} \\
\hline
\end{tabular}

Medias seguidas por la misma letra, mayúscula en la fila y minúscula en la columna, no difieren entre sí según la prueba de Tukey $(\mathrm{P} \leq 0,05)$

\section{CONCLUSIONES}

Cenchrus echinatus y Rottboellia exaltata interfieren negativamente en las características de crecimiento de plantas de frijol, y el efecto es mayor a medida que aumenta la densidad de la maleza.

Cenchrus echinatus fue la maleza que más ocasionó efecto negativo en las características de crecimiento de las plantas.

\section{LITERATURA CITADA}

1. Albuquerque, J.A., V.F. Melo, M.B. Soares, E.L. Finoto, R.H.S. Siqueira y S.A. Martins. 2013. Fitossociologia e características morfológicas de plantas daninhas após cultivo de milho em plantio convencional no cerrado de Roraima. Revista Agro@mbiente On-line 7(3): 313-321.

2. Alves, J.M., N.P. De Araújo, S.C. Uchôa, J.D. Albuquerque, A.J. Silva, G.S Rodrigues y D.C. Silva. 2009. Avaliação agroeconômica da produção de cultivares de feijão-caupi em consórcio com cultivares de mandioca em Roraima. Revista Agro@mbiente On-line 3(1): 15-30.

3. Castro, T.S., P.R. Rocha, G.F. Barreto, S.S. Maia, J.A. Albuquerque y J.M. Alves. 2019. Weed interference in semi-erect and semiprostate cowpea cultivars. Planta Daninha (37): e019196146.

4. CONAB. 2019. Companhia Nacional de Abastecimento. Acompanhamento da safra brasileira de grãos. v.6 Safra 2018/19 - Sétimo levantamento. pp. 1-119.

5. Cury, J.P., J.B. Santos, E.B. Silva, R.R. Braga, F.P. Carvalho, D. Valadão Silva y E.C. Byrro.
2013. Eficiência nutricional de cultivares de feijão em competência com plantas daninhas. Planta Daninha 31(1): 79-88.

6. Cury, J.P., J.B. Santos, D. Valadão Silva, F.P. Carvalho, R.R. Braga, E.C. Byrro y E.A. Ferreira. 2011. Produção e partição de matéria seca de cultivares de feijão em competencia com plantas daninhas. Planta daninha 29(1): 149-158.

7. Di Tomaso, J.M. 1995. Approaches for improving crop competitiveness through the manipulation of fertilization strategies. Weed Science 43(3): 491-497.

8. EMBRAPA Meio Norte. Estatística da produção de feijão-caupi. 2009. http://www.portaldoagronegocio.com.br/conteu do.php?id=3 4241 (consulta de marzo 3, 2019).

9. Ferreira, D.F. 2011. Sisvar: a computer statistical analysis system. Ciência e Agrotecnologia 35(6): 1039-1042.

10.Forte, C.T., F.J., Basso, L. Galon, L.R. Agazzi, F. Nonemacher y G. Concenço. 2017. Habilidade competitiva de cultivares de soja transgênica convivendo com plantas daninhas. Revista Brasileira de Ciências Agrárias 12(2): 185-193.

11.Freitas, F.C., V.F. Medeiros, L.C. Grangeiro, M.G. Silva, P.G. Nascimento y G.H. Nunes. 2009. Interferência de plantas daninhas na cultura do feijão-caupi. Planta Daninha 27(2): 241-247.

12.Lage, P., M.A. Silveira Júnior, E.A. Ferreira, G.A. Pereira y E.B. Silva. 2017. Interferência do arranjo de plantas daninhas no crescimento do feijoeiro. Revista de Agricultura Neotropical 4(3): 61-68.

13.Lamego, F.P., C.J. Basso, R.A. Vidal, M.M. 
Trezzi, A.L. Santi, Q. Ruchel et al. 2011. Seletividade dos herbicidas smetolachlor e alachlor para o feijão-carioca. Planta Daninha (29): 877-883.

14.Manabe, P.M., C.C. Matos, E.A. Ferreira, A.A. Silva, T. Sediyama, A. Manabe et al. 2014. Características fisiológicas de feijoeiro em competição com plantas daninhas. Bioscience Journal 30(6): 1721-1728.

15.Manabe, P.M., C.C. Matos, E.A. Ferreira, A.F. Silva, A.A. Silva, T. Sediyama et al. 2015. Efeito da competição de plantas daninhas na cultura do feijoeiro. Bioscience Journal 31(2): 333-343.

16. Novais, R.F. 2002. Um sistema simples de interpretação de análise de solo e recomendação de corretivos e fertilizantes. FertBio, Santa Maria (RS). https:// docplayer.com.br/8337114-Um-sistema-simple s-de-interpretacao-de-analise-de-solo-e-recome ndacao-de-corretivos-e-fertilizantes-1-rober toferreira-de-novais.html (consulta de marzo 3, 2019).

17.Oliveira, O.M., J.F. Silva, J.R. Gonçalves y C.S. Klehn. 2010. Período de infestacion das plantas daninhas com cultivares de feijão-caupi em várzeas no Amazonas. Planta daninha 28(3): 523-530.

18.Oliveira, S.R., A.S. Andrade Júnior, V.Q. Ribeiro, R.R. Brito y M.W. Carvalho. 2015.
Interação de níveis de água e densidade de plantas no crescimento e produtividade do Feijão-Caupi, em Teresina, PI. Irriga 20(3): 502-513.

19.Parreira, M.C., P.L. Alves y L.A. PeñaherreraColina. 2011. Influencia de las malezas sobre el cultivo de frijol en función de espaciamiento y de la densidad de plantas. Planta Daninha 29(4): 761-769.

20.Pessôa, U.C., E.N. Terceiro, A.S. Souza, A.A. Filho y T.A. Pimenta. 2015. Interferência de tiririca (Cyperus rotundus L.) e da compactação do solo no crescimento do feijãocaupi. Revista Verde 10(5): 61-69.

21.Pitelli, R.A. 1985. Interferência de plantas daninhas em culturas agrícolas. Informe Agropecuário 11(127): 16-27.

22.Silva, G.C., R.C. Magalhães, A.C. Sobreira, R. Schmitz y L.C. Silva. 2016. Rendimento de grãos secos e componentes de produção de genótipos de feijão-caupi em cultivo irrigado e de sequeiro. Revista Agro@mbiente On-line 10(4): 342-350.

23.Terceiro, E.N., U.C. Pessôa, A.S. Souza, A.A. Filho y T.A. Pimenta. 2016. Aspectos fisiológicos do feijão-caupi e crescimento de tiririca (Cyperus rotundus L.) sob competição em solo compactado. Revista Verde de Agroecologia e Desenvolvimento Sustentável 11(1): 14-22. 